\title{
DETERMINAÇÃO DA ENTALPIA DE VAPORIZAÇÃO DE LÍQUIDOS PELO MÉTODO DO ISOTENISCÓPIO DE SMITH E MENZIES
}

\author{
Adriana Passarella Gerola, Lilian Somenci Peloi, Noboru Hioka e Ourides Santin Filho* \\ Departamento de Química, Centro de Ciências Exatas, Universidade Estadual de Maringá, Av. Colombo, 5790, 87020-900 Maringá \\ - PR, Brasil \\ Lilian Akemi Kato \\ Departamento de Matemática, Centro de Ciências Exatas, Universidade Estadual de Maringá, Av. Colombo, 5790, 87020-900 \\ Maringá - PR, Brasil
}

Recebido em 30/10/08; aceito em 30/7/09; publicado na web em 11/1/10

\begin{abstract}
DETERMINATION OF THE ENTHALPY OF VAPORIZATION OF LIQUID COMPOUNDS BY THE SMITH MENZIES (ISOTENISCOPE) METHOD. This article proposes an experimental procedure to determine the enthalpy (and entropy) of vaporization of organic liquid compounds, by the Smith-Menzies (isoteniscope) method. The values of vapor pressure at different temperatures were obtained and $\Delta_{\mathrm{v}} \mathrm{H}\left(\right.$ and $\left.\Delta_{\mathrm{v}} \mathrm{S}\right)$ were graphically determined, using the Clausius-Clapeyron equation. The results for diethyl-ether, propanone, ethanol and n-hexane are in very good agreement with those from literature. A historical and thermodynamic discussion on equations that correlates vapor pressures and temperature precedes the experimental proposition.
\end{abstract}

Keywords: enthalpy of vaporization; isoteniscope; organic compounds.

\section{INTRODUÇÃO}

Quando um líquido é colocado em um recipiente fechado e sob vácuo, suas moléculas escapam da fase líquida para a fase gasosa até que se atinja um equilíbrio a uma dada temperatura. Esse equilíbrio é dinâmico, com moléculas passando do estado vapor ao estado líquido e vice-versa. A pressão de vapor é definida como a pressão exercida por esse vapor em equilíbrio com o líquido a essa dada temperatura. ${ }^{1}$

A pressão de vapor e a temperatura são diretamente proporcionais de modo que, com o aumento da temperatura, há maior quantidade de energia disponível para romper as forças que sustentam as moléculas unidas no estado líquido. Como consequência, haverá maior quantidade de vapor no recipiente, que se traduz no aumento da pressão de vapor.

A relação de dependência entre a pressão de vapor e a temperatura pode ser representada por meio da Equação de Clausius-Clapeyron (1):

$\ln \frac{p_{\text {vap }}}{p^{0}}=\frac{-\Delta_{\text {vap }} H}{R} \cdot \frac{1}{T}+c t e$

onde $p_{\text {vap }}$ representa a pressão de vapor do composto, $p^{0}$ é a pressão padrão, $\Delta_{v a p} H$, é a entalpia de vaporização, $R$ é a constante dos gases ideais e $T$ é a temperatura absoluta do sistema.

A entalpia de vaporização corresponde ao calor de vaporização à pressão constante e tem valor sempre positivo, visto que uma mudança de fase do estado líquido para vapor requer fornecimento de energia (o processo é endotérmico). O valor de $\Delta_{\text {vap }} H$ permite avaliar a intensidade das interações atrativas existentes no líquido. Tal mudança implica em aumento significativo da distância média molecular e do grau de desordem, com consequente aumento da entropia do sistema. A diferença entre a entropia da fase de vapor e a entropia da fase líquida caracteriza a entropia de vaporização $\left(\Delta_{v a p} S\right) .^{1,2}$

Inúmeros líquidos orgânicos apresentam entropia-padrão de vaporização (aumento da entropia na passagem do estado líquido ao de vapor no ponto de ebulição normal do líquido, $\Delta_{v a p} S^{\circ}$ ), de aproxi-

*e-mail: osantin@uem.br madamente $85 \mathrm{~J} \mathrm{~K}^{-1} \mathrm{~mol}^{-1}$, isto é, obedecem a chamada regra empírica de Trouton, proposta em 1884 por Frederick Thomas Trouton. ${ }^{3}$ A explicação para esta regra é que aproximadamente o mesmo aumento na desordem ocorre quando qualquer líquido se converte em vapor, de modo que a variação de entropia é independente, a princípio, da natureza do líquido. Desvios da regra de Trouton ocorrem para líquidos fortemente associados. Por exemplo, devido às ligações de hidrogênio intermoleculares, a entropia de vaporização da água é de $109 \mathrm{~J} \mathrm{~K}^{-1} \mathrm{~mol}^{-1}$ a $100{ }^{\circ} \mathrm{C}$, enquanto que para o ciclo-hexano o valor acompanha a regra de Trouton plenamente $\left(85 \mathrm{~J} \mathrm{~K}^{-1} \mathrm{~mol}^{-1}\right){ }^{4}$

Neste trabalho propõe-se uma atividade experimental de determinação da entalpia de vaporização de líquidos orgânicos a partir de medidas de seus valores de pressão de vapor em diferentes temperaturas, adotando-se o método do isoteniscópio, também conhecido como método de Smith e Menzies. ${ }^{5,6} \mathrm{~A}$ base deste método é o princípio da igualdade da pressão sobre os dois meniscos, mantidos na mesma altura, de um líquido contido em um tubo em formato de "U", no qual uma das extremidades está fechada e saturada de vapor do líquido, enquanto que a outra se comunica com um manômetro de leitura. A partir dos resultados da pressão de vapor do lado fechado do isoteniscópio em função da temperatura, a entalpia e a entropia de vaporização são determinadas graficamente, recorrendo-se à Equação de Clausius-Clapeyron.

Embora não seja original, o experimento é pouco conhecido. Além de simples e altamente didático, requer aparelhagem de custo relativamente reduzido, o que viabiliza seu uso nas mais diversas instituições brasileiras onde cursos de Química são ministrados.

\section{Contexto histórico}

Fenômenos de mudança de fase são descritos e conhecidos desde tempos muito antigos. Na Grécia Antiga, Tales de Mileto (séc. VII ou VI a.C.) supunha que a physis, palavra associada ao termo moderno natureza, tinha como única origem ou princípio a água. Esta passaria por transformações que, no sentido atual, incluiriam a passagem líquido-vapor na geração dos demais corpos. Sobre esse fato comenta o filósofo Heráclito, 
... a substância natural úmida, uma vez que facilmente se transforma em cada uma das diferentes coisas, está acostumada a passar por variadíssimas modificações: a parte dela que se exala transforma-se em ar, e a parte mais sutil inflama-se de ar em éter, ao passo que a água, quando se torna compacta e se transforma em lodo, converte-se em terra. Por isso Tales declarou que, dos quatro elementos, a água era, por assim dizer, o mais ativo, enquanto causa (dos demais). ${ }^{7}$

Nota-se nesse trecho a possibilidade de transformação da água em ar, fenômeno que então incluía os processos de vaporização.

Para Aristóteles de Estagira (384-322 a.C.), o mundo sublunar (abaixo da Lua) seria constituído por quatro elementos (água, terra, fogo e ar), transformáveis uns nos outros. De acordo com o estagirita, "de fato, as mais essenciais diferenças entre os corpos são as diferenças de qualidades, funções e potências". ${ }^{8}$ Ora, para Aristóteles a água possuía as qualidades de ser úmida e fria, e o ar possuía as qualidades de ser úmido e quente. Transformar a água em ar significa, então, transferir calor para a água a fim de convertê-la de úmida e fria em úmida e quente. $\mathrm{O}$ fogo concorre para este evento, transformando-a em vapor, que se dissipa na atmosfera.

Modernamente, as primeiras medidas controladas da pressão de vapor de líquidos puros em função da temperatura que se tem notícia são devidas ao meteorologista inglês John Dalton (1766-1844). Segundo Wisniak, ${ }^{9}$ ele estudou o comportamento da pressão em função da temperatura para os seguintes líquidos: água, éter, espírito de vinho (álcool), amônia, mercúrio e ácido sulfúrico. Dalton observou que a pressão de vapor aumentava em progressão geométrica, enquanto que a temperatura aumentava em progressão aritmética, e formulou a seguinte Equação, em que a temperatura aparece ainda na escala Celsius:

$P=P_{0} \cdot a^{t}$

em que $a$ é uma constante.

A Equação 2 pode ser transformada em

$\log P=b+c . t$

onde temos as seguintes constantes $b=\log P_{0}$ e $c=\log a$

Jean Baptiste Biot (1774-1862), físico francês e pioneiro nos estudos de polarimetria, modificou a Equação de Dalton, dando-lhe a forma

$\log P=a+b \alpha^{t}+c \beta^{t}$

em que $a, b, c$, $\alpha$ e $\beta$ são parâmetros a serem determinados experimentalmente, com a temperatura ainda na escala Celsius.

Victor Regnault (1810-1878), estudioso das propriedades térmicas dos gases, introduziu novo valor de referência para escala de temperatura em Celsius, e modificou a Equação de Biot para

$\log P=a+b \alpha^{\theta}+c \beta^{\theta}$

O parâmetro $\theta$ corresponde à temperatura medida a partir de algum valor de referência (zero) arbitrário, por exemplo, a menor das temperaturas de alguma série de experimentos utilizada para se determinar o valor das constantes. Em termos de escala Celsius pode-se escrever $\theta=t+n$.

Carlo Amedeo Avogadro (1776-1856), propositor da hipótese molecular dos gases, determinou a pressão de vapor do mercúrio em temperaturas muito próximas de seu ponto de ebulição, tendo trabalhado até cerca de $300{ }^{\circ} \mathrm{C}$, limite superior das escalas termométricas de mercúrio disponíveis para a época. Avogadro fez uso de um sifão de vidro invertido e conectado a um balão imerso em óleo de oliva. Na prática, ele fez uso de um isoteniscópio, cuja técnica se constitui no tema deste trabalho. Avogadro deduziu a relação abaixo: ${ }^{10}$

$T_{r e l}=P(1-l / L)$

onde $T_{\text {rel }}$ corresponde a uma medida da temperatura relativa no sentido em que $T_{r e l}=0$ é a temperatura quando, a uma determinada pressão, a altura $l$ do lado fechado do isoteniscópio alcança a altura $L$, correspondente ao lado aberto do mesmo. Avogadro modificou sua equação para

$P=(1+a t)^{m}$

na qual $P$ é a pressão de vapor em atm e $t$ é a temperatura relativa à temperatura de ebulição do mercúrio, em unidades de $100^{\circ} \mathrm{C}, \operatorname{com} a \mathrm{e}$ $m$ a serem determinados. O cientista constatou que a Equação 7 produz resultados muito satisfatórios entre 230 e $300{ }^{\circ} \mathrm{C}$. Para temperaturas menores, ele propôs uma Equação de Biot modificada

$\log P=a t+b t^{2}+c t^{3}$

com $a, b$ e $c$ dados por $a=-0,64637 ; b=0,075956$ e $c=0,18452$.

A dificuldade em se determinar a pressão de vapor de líquidos a temperaturas muito inferiores ao seu ponto de ebulição fica explicitada nessa equação, pois Avogadro obteve o valor de $\mathrm{P}=8,498 \mathrm{x}$ $10^{-8} \mathrm{mmHg}$ para o mercúrio a $\mathrm{t}=0{ }^{\circ} \mathrm{C}$, quando seu valor é $\mathrm{P}=1,85$ x $10^{-5} \mathrm{mmHg} .{ }^{11}$

Jacques Louis Bertrand (1822-1900) partiu, por sua vez, de uma equação bastante próxima àquela de Clausius-Clapeyron na sua forma diferencial, ${ }^{12}$

$\frac{d P}{d T}=\frac{\Delta_{v a p} H}{A T V}$

na qual $A$ é uma constante e $V$ é o volume molar do vapor. A partir dessa equação, e dependendo da relação encontrada entre $P, T$ e $V$, Bertrand sugeriu três equações integradas, para diferentes sistemas

a) Para a água, $P=k \cdot \frac{T^{\alpha}}{(T+a)^{\beta}}$

na qual $\alpha=79,623 ; \beta=88,623$ e $a=126,37$.

b) Para diversas substâncias, na qual o fator $\phi=\frac{A P V}{\Delta_{\text {vap }} H}$ depende
pouco da temperatura, tal que $P=k T^{n}$

$\operatorname{com} n=1 / \phi$.

c) Para outras substâncias, em que $\phi=a T-b$, tal que

$P=G\left[\frac{T-\lambda}{T}\right]^{n}$

em que $\mathrm{G}=$ constante e $n=1 / b$ e $\lambda=b / a$.

\section{A Equação de Clapeyron}

Benoit-Pierre Émile Clapeyron nasceu em Paris no ano de 1799. Foi engenheiro e matemático, especialista em construção de locomotivas e ferrovias. Especializando-se em projetos de locomotivas a vapor, foi para a Inglaterra (1836) procurar Stevenson, o mais famoso dos 
construtores de locomotivas da época, mas este achou os seus projetos muito complicados. Desestimulado na Inglaterra, voltou à França, onde estendeu suas atividades para projetos de pontes metálicas. Em Paris, foi nomeado professor da École des Ponts et Chaussées (1844) e eleito para a Academia de Ciências (1848), onde serviu em muitos comitês, em particular no comitê para premiações em mecânica. Também serviu em um comitê de acompanhamento da construção do Canal de Suez e em outro que analisou o emprego de máquinas a vapor na marinha. Sua mais importante contribuição científica foi a apresentação de uma análise matemática das ideias de Sadi Carnot sobre calor (1834), uma formulação matemática do conhecido ciclo de Carnot, dando-lhe a credibilidade científica final e tornando esse princípio fundamentado cientificamente. Sua equação diferencial para determinação do calor de vaporização de um líquido influenciou de modo fundamental o trabalho de Thomson e de Clausius na formulação do enunciado da segunda lei de termodinâmica. Clapeyron faleceu em 1864.

Partindo das ideias e do trabalho de Sadi Carnot, Clapeyron elaborou um trabalho (E. Clapeyron, Journal de l'École Polytechnique, v. 14, 1834, apud Wisniak) em que analisa o calor total recebido e perdido em dois ciclos de Carnot, o primeiro deles onde o sistema tem comportamento de gás ideal (portanto, acima do ponto crítico) e o segundo operando na região de líquido-vapor. Em ambos os casos foram abordadas isotermas bastante próximas entre si, separadas por um infinitésimo de temperatura $d T$ (Figura 1).

Tal aproximação permitiu tratar os ciclos de Carnot como paralelogramos e obter resultados de transferência de calor a partir de considerações geométricas.

Para um gás de comportamento ideal, Clapeyron adotou a aproximação

$$
p V=R(267+t), \operatorname{com} R=\frac{p_{0} V_{0}}{267+t_{0}}
$$

A Equação 10 mostra claramente que, além dele não ter usado a noção de temperatura absoluta, o zero de escala está localizado em $-267^{\circ} \mathrm{C}$
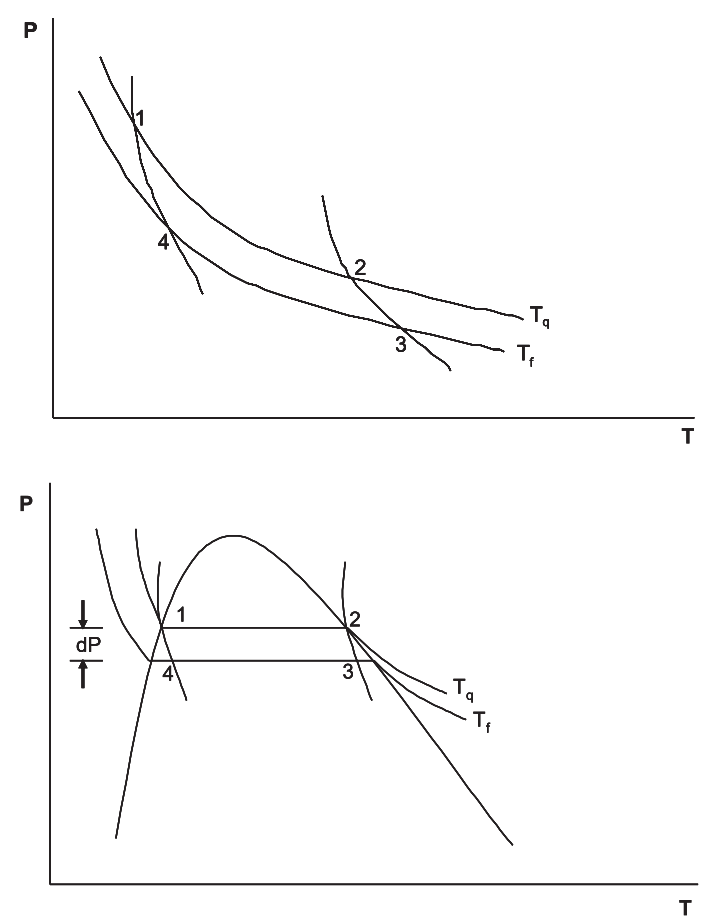

Figura 1. a. Ciclo de Carnot em região afastada do ponto crítico; b. ciclo de Carnot em região vizinha ao ponto crítico
A aproximação da região delimitada pelos pontos 1, 2, 3 e 4 no gráfico da Figura 1a por um paralelogramo permitiu a Clapeyron determinar a Equação 11 para a transferência de calor da fonte quente para a fonte fria

$Q=R(B+C \log P)$

na qual $B$ e $C$ são constantes indeterminadas que dependem da temperatura. Segundo Wisniak, ${ }^{12}$ a Equação 11 equivale à seguinte equação moderna

$T d S=C_{P} d T-T\left(\frac{\partial V}{\partial T}\right)_{P} d P$

Para o ciclo na região de líquido-vapor, o resultado obtido por Clapeyron foi

$k=C\left(1-\frac{V_{L}}{V_{G}}\right) \frac{d P}{d T}$

onde $k$ corresponde ao calor latente de vaporização por unidade de volume de vapor.

\section{A Equação de Clausius-Clapeyron}

Rudolf Clausius nasceu em 2/1/1822 em Koslin, Prússia. Seu pai foi pastor, professor e conselheiro no Royal Government School Board, tendo fundado uma pequena escola privada em que Rudolf adquiriu a sua formação inicial. Clausius ingressou na Universidade de Berlim em 1840 e lá estudou matemática e física. Formou-se em 1844 e dois anos depois apresentou na Universidade de Halle a sua tese de doutoramento, que foi aprovada com distinção em 15/7/1848. Em 1855 tornou-se professor de matemática e física no Polytechnic Institute, em Zurique. Foi professor na Universidade de Würzburg (1865-1867) e em 1867 foi nomeado professor na Universidade de Bonn, onde assumiu a reitoria nos últimos anos da sua vida (1884-1888)

Rudolf Clausius foi um dos físicos mais notáveis do século XIX. O começo significativo de sua carreira data de 1850 , quando estabeleceu os fundamentos para a moderna termodinâmica no artigo Uber die bewegende Kraft der Wärme, apresentado à Academia de Berlim a 18 de Outubro daquele ano. Suas contribuições mais importantes para o desenvolvimento da Física foram a formulação da segunda lei da termodinâmica e o desenvolvimento da teoria mecânica do calor sobre as bases do conceito de entropia. Clausius introduziu ainda algumas melhorias no tratamento matemático da primeira lei da termodinâmica e estudou a relação entre a teoria cinética e a termodinâmica, tentando interligar o ponto de vista microscópico com o macroscópico. Faleceu em Bonn, Alemanha, a $24 / 8 / 1888$

Tendo em mãos os trabalhos de Carnot e de Clapeyron, além de novos dados experimentais disponíveis, Clausius modificou a Equação 10, então conhecida como lei de Mariotte-Gay Lussac, para

$P V=\frac{P_{0} V_{0}}{273+t_{0}}(a+t)$

Como se pode ver na Equação 14, o zero na escala de temperatura absoluta já estava localizado em $t=-273{ }^{\circ} \mathrm{C}$.

Clausius concluiu que na Equação 13, de Clapeyron, a constante $C$ assume a forma $C=A(a+t)$, e reescreveu-a como

$r=A(a+t)\left(V_{G}-V_{L}\right) \frac{d P}{d t}$ 
com $r=$ calor latente de vaporização.

Em linguagem moderna, assumindo as hipóteses que:

$A(a+t)=T \quad$ e $\quad r=\Delta_{\text {vap }} H$

podemos escrever

$\Delta_{v a p} H=T \Delta V \frac{d P}{d T}$ rearranjando, $\frac{d P}{d T}=\frac{\Delta_{v a p} H}{T \Delta V}$

Considerando agora que o volume de um gás seja muito maior do que o do líquido, o que é razoável para sistemas não muito próximos do ponto crítico, e que a fase de vapor se comporta como gás ideal

$\frac{d P}{d T}=\frac{\Delta_{v a p} H}{R T^{2}} p \Rightarrow \frac{d P}{P} \frac{1}{d T}=\frac{\Delta_{v a p} H}{R T^{2}} \Rightarrow \frac{d \ln P}{d T}=\frac{\Delta_{v a p} H}{R T^{2}}$

A entalpia de vaporização se comporta de modo independente da temperatura para faixas não muito largas de $T$, podendo ser assumida como constante. Neste caso, a Equação 18 pode ser integrada em função de $T$, obtendo-se finalmente a Equação de Clausius-Clapeyron

$\ln \frac{P}{P^{0}}=\frac{-\Delta_{\text {vap }} H}{R T}+c$

O que resulta, para dois estados $P_{1}, T_{1}$ e $P_{2}, T_{2}$, em

$\ln \frac{P_{2}}{P_{1}}=\frac{\Delta_{\text {vap }} H}{R}\left(\frac{1}{T_{1}}-\frac{1}{T_{2}}\right)$

\section{A equação de Clausius-Clapeyron nos livros-textos modernos}

Deduções clássicas ${ }^{13}$ da Equação de Clapeyron partem da condição de equilíbrio entre duas fases para uma substância pura e da igualdade de seus potenciais químicos, i.e., dado o equilíbrio líquido-vapor,

$X_{L} \leftrightarrow X_{V}$

os potenciais químicos são iguais,

$\mu_{L}=\mu_{V}(P, T=$ constantes $)$

Pequenas alterações de pressão e temperatura conduzem a pequenas alterações nos potenciais químicos, idênticas para as duas fases

$\mu_{L}+d \mu_{L}=\mu_{V}+d \mu_{V}$

$(T+d T, P+d P)(22)$

o que implica em $d \mu_{L}=d \mu_{V}$

A partir da Equação de Gibbs-Duhem, pode-se então escrever,

$\bar{V}_{L} d P-\bar{S}_{L} d T=\bar{V}_{V} d P-\bar{S}_{V} d T$

$\bar{S}_{V} d T-\bar{S}_{L} d T=\bar{V}_{V} d P-\bar{V}_{L} d P$

$\left(\bar{S}_{V}-\bar{S}_{L}\right) d T=\left(\bar{V}_{V}-\bar{V}_{L}\right) d P$

$\frac{\mathrm{dP}}{\mathrm{dT}}=\frac{\Delta_{\text {vap }} \overline{\mathrm{S}}}{\Delta \overline{\mathrm{V}}}$

Para as duas fases em equilíbrio $\Delta G=\Delta H-T \Delta S=0$, o que implica em $\Delta S=\Delta H / T$, então

$\frac{\mathrm{dP}}{\mathrm{dT}}=\frac{\Delta_{\text {vap }} \overline{\mathrm{H}}}{\mathrm{T} \Delta \overline{\mathrm{V}}}$ Equação de Clapeyron
Vale notar que a Equação de Clapeyron fornece a taxa de variação da pressão com a temperatura em torno de uma dada temperatura $T$ de equilíbrio entre duas fases de uma substância pura. Dito de outra forma, a equação dá a tangente em cada ponto das curvas de transição de fase num diagrama $P V$. A integração da Equação de Clapeyron fornece, então, a equação das linhas de fronteira entre as diversas fases.

Opção de dedução equivalente parte da relação de Maxwell, em particular a que correlaciona as variações de entropia com variações de temperatura e volume

$T d S=C_{V} d T+T\left(\frac{\partial P}{\partial T}\right)_{V} d V$

Considerando que as transições de fase ocorrem a temperatura constante, $d T=0, \log 0$

$T d S=T\left(\frac{\partial P}{\partial T}\right)_{V} d V$

que, por fim, se reduz à Equação 24 pelas mesmas considerações já feitas.

A Equação de Clausius-Clapeyron (1) pode ser também facilmente deduzida da Equação de Van't Hoff (28), supondo o volume do líquido ou do sólido desprezível em relação à variação de volume do vapor e considerando-o como um gás perfeito.

$\frac{d \ln K_{P}}{d T}=\frac{\Delta_{v a p} H^{0}}{R T^{2}}$

Para uma transformação da fase líquida para gasosa, o valor de $K_{P}$ é igual à pressão de vapor, $P_{v a p}$, logo, a Equação de ClausiusClapeyron é obtida pela substituição de $K_{P}$ na Equação 28. Esta analogia serve também para mostrar que o calor latente nada mais é do que o calor de reação na ausência de transformação química. ${ }^{14}$

Uma alternativa interessante é fornecida por Igor Novak. ${ }^{15} \mathrm{O}$ autor considera a natureza dinâmica dos equilíbrios químicos, evidenciando as relações entre cinética e termodinâmica.

Consideremos um líquido puro em equilíbrio com seu vapor,

$X_{L} \leftrightarrow X_{V}$

A constante de equilíbrio do processo acimaé dada pela razão entre as constantes de equilíbrio de vaporização $\left(k_{V}\right)$ e de condensação $\left(k_{C}\right)$, tal que $K=\frac{k_{V}}{k_{C}}$

As Equações de Arrhenius para os dois processos são

$$
\begin{aligned}
& \ln k_{V}=\ln A_{V}-\frac{E_{a, V}}{R T} \\
& \ln k_{C}=\ln A_{C}-\frac{E_{a, C}}{R T}
\end{aligned}
$$

Subtraindo uma equação da outra

$$
\begin{aligned}
& \ln k_{V}-\ln k_{C}=\ln A_{V}-\ln A_{C}-\frac{\left(E_{a, V}-E_{a, C}\right)}{R T} \\
& \ln \frac{k_{V}}{k_{C}}=\ln K=C_{1}-\frac{\left(E_{a, V}-E_{a, C}\right)}{R T}
\end{aligned}
$$


a constante $C_{1}$ refere-se à diferença entre os logaritmos das constantes de Arrhenius.

As energias de ativação para a vaporização $\left(E_{a, V}\right)$ e para a condensação $\left(E_{a, C}\right)$ podem ser escritas em termos de energia interna $(U)$, conforme a Figura 2.

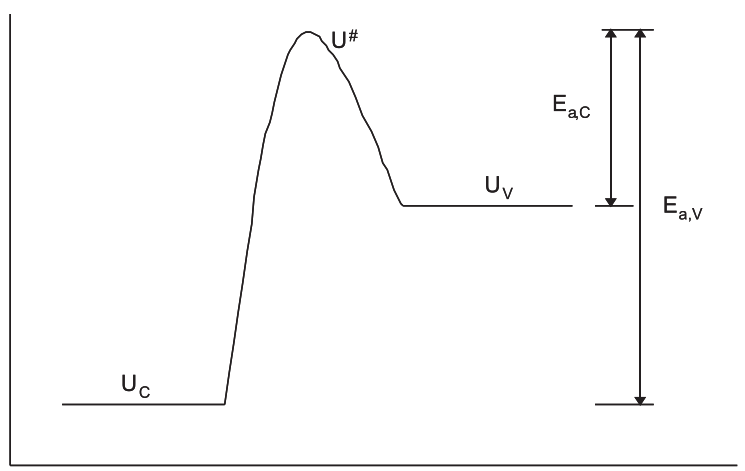

Figura 2. Diagrama de energia versus coordenada de mudança de fase líquido/vapor

A partir da Figura 2 pode-se escrever

$E_{a, V}=E_{a, C}+\left(U_{V}-U_{C}\right)$

tal que $U_{V C C}$ são as energias internas das fases vapor e condensada, respectivamente. Então,

$E_{a, V}-E_{a, C}=U_{V}-U_{C}$

A partir da definição de entalpia, valem as relações abaixo para cada uma das fases

$U_{C}=H_{C}$

$U_{V}=H_{V}-(P V)_{V}$

Logo,

$E_{a, V}-E_{a, C}=H_{V}-(P V)_{V}-H_{C}$

$E_{a, V}-E_{a, C}=\Delta_{v a p} H_{\sharp}-(P V)_{V}$

Assumindo comportamento de gás ideal, tal que $P V=n R T=$ constante e substituindo (35b) em (32),

$\ln \frac{k_{V}}{k_{C}}=\ln K=C_{1}-\frac{\Delta_{\text {vap }} H-n R T}{R T}$

$\ln K=C_{1}-\frac{\Delta_{v a p} H}{R T}-\frac{n R T}{R T}$

$\ln K=C_{1}-n-\frac{\Delta_{v a p} H}{R T}$

$\ln K=C_{2}-\frac{\Delta_{\text {vap }} H}{R T}$

Como a constante de equilíbrio $(K)$ é proporcional à pressão de vapor $P$ do sistema, pode-se escrever $\ln K=\ln P+C_{3}$, chegando-se, por fim, à Equação de Clausius-Clapeyron $\ln P+C_{3}=C_{2}-\frac{\Delta_{v a p} H}{R T} \rightarrow \ln P=C_{2}-C_{3}-\frac{\Delta_{v a p} H}{R T}$

$\ln P=C_{4}-\frac{\Delta_{v a p} H}{R T}$ (Equação de Clausius-Clapeyron)

\section{PARTE EXPERIMENTAL}

Foram utilizados os seguintes líquidos orgânicos, com suas procedências: álcool etílico absoluto (Dinâmica), acetona PA (Nuclear), éter etílico PA (Chemco) e hexano PA (Nuclear). Uma vez que a purificação requer custos e condições nem sempre disponíveis nas escolas brasileiras, resolvemos utilizar os materiais na condição em que foram adquiridos.

Para a montagem do arranjo experimental foram utilizados um manômetro de mercúrio convencional, um termômetro, um barômetro, uma chapa de aquecimento com agitação magnética, um béquer de $1 \mathrm{~L}$, suporte universal, garras metálicas, mangueiras (de preferência de silicone, menos suscetível de reagir com os vapores orgânicos), um erlenmeyer de segurança posicionado após o manômetro, uma bomba de vácuo com controle nas saídas de vácuo e pressão, um frasco "Dewar" (garrafa térmica), um "dedo frio" e um isoteniscópio (neste caso constituído apenas por um tubo de vidro fechado em um dos lados, dobrado em formato de "U").

A Figura 3 mostra um diagrama do arranjo experimental usado no laboratório. A saída do isoteniscópio comunica-se com uma junção em "T" com saídas simultâneas para um manômetro e uma bomba de vácuo. A torneira "A" (opcional) permite isolar o manômetro do resto do circuito e deve permanecer aberta durante a execução das medidas. Por segurança, a saída do manômetro para a atmosfera é conectada a uma mangueira com extremidade presa e inserida em um erlenmeyer contendo um pouco de algodão. Desse modo, caso acidentalmente ocorra aumento brusco de pressão no circuito, evita-se o derramamento de mercúrio para o ambiente do laboratório.

No percurso entre o isoteniscópio e a bomba de vácuo instala-se ainda um sifão de segurança (dedo frio) que pode ser resfriado com nitrogênio líquido ou, na falta deste, misturas refrigerantes, tais como acetona/ álcool com gelo seco, ${ }^{16}$ no interior de um frasco de Dewar. Esse recurso impede que vapores orgânicos alcancem o interior da bomba. Cuidados extras devem ser tomados com relação à possibilidade de entupimento no dedo frio pela condensação de quantidades excessivas do composto.

O sistema deve ser cuidadosamente montado, evitando-se o contato das borrachas de conexão com o béquer aquecido e com a chapa térmica. Outro cuidado importante a ser tomado é com as etapas de sucção/compressão de ar no sistema, que devem sempre ser conduzidas de modo suave a fim de se evitar variações bruscas de pressão no circuito manômetro/isoteniscópio.

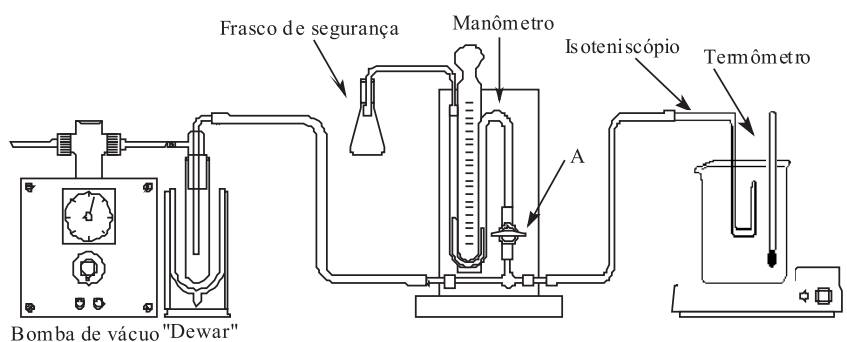

Figura 3. Arranjo experimental para determinação da pressão de vapor de líquidos em diversas temperaturas 
Tabela 1. Valores de pressão de vapor obtidos em diversas temperaturas, para os quatro compostos orgânicos estudados

\begin{tabular}{|c|c|c|c|c|c|c|c|c|c|c|}
\hline \multirow{2}{*}{ Éter etílico } & $T / K$ & 298,5 & 301,2 & 312,0 & 314,5 & 317,5 & & & & \\
\hline & $\mathrm{p} / \mathrm{mmHg}$ & 539,2 & 595,9 & 880,1 & 943,9 & 1064,2 & & & & \\
\hline \multirow{2}{*}{ Acetona } & $T / K$ & 309,6 & 313,5 & 315,5 & 323,6 & 325,9 & 328,1 & 330,0 & & \\
\hline & $\mathrm{p} / \mathrm{mmHg}$ & 474,9 & 542,9 & 603,0 & 805,9 & 870,0 & 955,0 & 1013,9 & & \\
\hline \multirow{2}{*}{ Etanol } & $T / K$ & 325,2 & 329,5 & 331,3 & 333,9 & 337,2 & 344,0 & 347,0 & 351,0 & 352,0 \\
\hline & $\mathrm{p} / \mathrm{mmHg}$ & 329,0 & 403,0 & 457,1 & 522,2 & 595,9 & 800,3 & 873,9 & 1030,7 & 1058,9 \\
\hline \multirow{2}{*}{ n-Hexano } & $T / K$ & 302,0 & 309,3 & 313,0 & 317,6 & 327,0 & 330,0 & 335,5 & 337,0 & 338,9 \\
\hline & $\mathrm{p} / \mathrm{mmHg}$ & 303,1 & 476,8 & 574,2 & 637,8 & 801,9 & 910,5 & 1024,5 & 1033,8 & 1090,1 \\
\hline
\end{tabular}

A homogeneização da temperatura do banho é obtida graças à agitação. Deve-se então tomar especial cuidado em se evitar o contato da barra magnética do agitador com a base do termômetro e do isoteniscópio.

Líquidos orgânicos são capazes de dissolver os gases atmosféricos em quantidade significativa. Desse modo, antes de se executar o experimento é importante que estes sejam removidos. Para tanto, introduz-se certo volume de líquido no isoteniscópio, tomando-se o cuidado de deixar uma pequena bolha de ar ( $3 \mathrm{~mm}$ de diâmetro) no lado fechado do mesmo. $\mathrm{O}$ volume de líquido acrescentado deve ser tal que o menisco do lado aberto fique de $3 \mathrm{a} 4 \mathrm{~cm}$ abaixo do menisco do lado fechado, que contém a pequena bolha de ar. A seguir, conecta-se o isoteniscópio no circuito, mantendo-o ainda fora do banho. Com o sifão frio montado e refrigerado, abre-se totalmente a válvula de escape do circuito de sucção da bomba de vácuo e a mesma é ligada. Nessa condição, a válvula de escape é lentamente fechada (até produzir uma diferença de altura dos meniscos de 10 a $15 \mathrm{~cm}$ ); observa-se um aumento de volume da bolha provocado pela redução da pressão aplicada sobre este com a incorporação de vapor do composto. A seguir, inclinando-se adequadamente o tubo isoteniscópio, aplicam-se pequenos golpes no mesmo de modo a estourar a bolha, permitindo que simultaneamente parte do vapor escape pela extremidade aberta do tubo mantendo-se, no entanto, uma pequena bolha na extremidade fechada. O processo deve ser repetido inúmeras vezes, sempre deixando uma pequena bolha remanescente que a todo momento irá aumentar de volume com o processo de succção. Neste processo a bolha irá se enriquecer de vapor do líquido orgânico diminuindo o teor de ar na mesma, até sua eliminação. Recomenda-se aplicar os golpes em um ritmo constante, evitando interrupções.

A determinação dos valores da pressão de vapor do líquido-problema é feita em diversas temperaturas, a partir do valor ambiente, sempre com o isoteniscópio mantido no interior do banho e sob agitação.

Após o sistema alcançar equilíbrio térmico, faz-se ajuste da pressão do sistema por meio da válvula de escape da bomba de vácuo, buscando igualar a altura dos dois meniscos no isoteniscópio. Alcançada esta condição, a pressão pode ser lida no manômetro. Em seguida, aumenta-se a temperatura do banho por cerca de 3 ${ }^{\circ} \mathrm{C}$, aguarda-se o equilíbrio térmico do sistema, faz-se novamente o ajuste da pressão buscando o nivelamento dos meniscos e efetua-se a leitura do manômetro. Este processo pode ser repetido até que se alcance a temperatura normal de ebulição do líquido nas condições do ambiente, ocasião em que a pressão no lado fechado do isoteniscópio equivale à pressão atmosférica local. A partir desse ponto, aumentos de temperatura farão com que a pressão de vapor do líquido supere a pressão atmosférica, de tal modo que a igualdade de altura dos meniscos somente será conseguida usando-se o sistema de compressão da bomba de vácuo. A partir desse ponto repete-se o ciclo de medidas agora usando compressão de ar.

\section{RESULTADOS E DISCUSSÃO}

A Tabela 1 contém os valores de pressão de vapor obtidos para quatro compostos orgânicos em diversas temperaturas.
Os dois primeiros compostos têm ponto de ebulição mais próximos da temperatura ambiente e sua alta volatilidade dificulta seu manuseio no sistema montado, daí o fato de terem sido coletados menos pontos para os mesmos.

Conforme discutido anteriormente, a pressão do vapor do líquidoproblema é determinada a partir da igualdade na altura dos meniscos do ramo aberto e fechado (vapor puro) do isoteniscópio. A partir dos valores de pressão de vapor $P_{v a p}$ de uma das substâncias (no caso a acetona), obtidos para cada temperatura elaborou-se o gráfico da Figura 4, que corresponde aos valores do logaritmo da pressão em função do recíproco da temperatura absoluta.

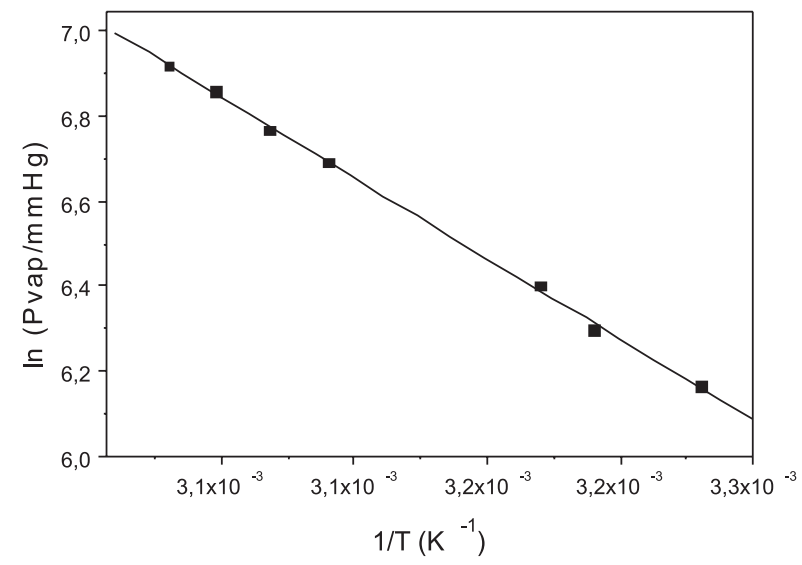

Figura 4. Valores de ln $P_{\text {vap }}$ versus $1 /$ T para a acetona

Gráficos similares foram elaborados para as demais amostras e suas linearidades podem ser avaliadas pelos seus coeficientes de correlação. A partir da Equação de Clausius-Clapeyron, determinouse a entalpia de vaporização para os quatro líquidos analisados, por meio do valor do coeficiente angular das retas obtidas em cada caso, que corresponde a $-\Delta_{v a p} H / R$. Os resultados encontram-se na Tabela 2 , juntamente com os desvios obtidos, adotando-se o desvio de cada medida como sendo metade da menor unidade da escala lida no termômetro e no manômetro a óleo. Os desvios finais foram obtidos a partir do tratamento estatístico de dados do programa gráfico.

É importante ressaltar que os valores de $\Delta_{\text {vap }} H_{\text {lit }}$ na Tabela 2 foram calculados a partir de valores de pressão em diversas temperaturas disponíveis na literatura.

Tabela 2. Valores experimentais e valores obtidos na literatura de entalpia molar de vaporização. A tabela mostra também as diferenças percentuais entre ambos e os coeficientes de correlação obtidos a partir das retas experimentais

\begin{tabular}{lcccc}
\hline Amostra & $\Delta_{\text {vap }} H_{\text {exp }} / \mathrm{kJ} \mathrm{mol}^{-1}$ & $\Delta_{\text {vap }} H_{l i t} / \mathrm{kJ} \mathrm{mol}^{-1 *}$ & $\Delta(\%)$ & $\mathrm{cc}$ \\
\hline Éter etílico & $28,0 \pm 0,4$ & 28,9 & 3,1 & 0,9994 \\
Acetona & $31,7 \pm 0,4$ & 31,2 & $-1,6$ & 0,9990 \\
Etanol & $41,2 \pm 0,4$ & 41,4 & 0,5 & 0,9966 \\
Hexano & $27,1 \pm 0,4$ & 31,5 & -14 & 0,9685 \\
\hline
\end{tabular}


Tabela 3. Valores de temperatura de ebulição normal $\left(T_{e b}\right)$ e entropias de vaporização para as quatro substâncias examinadas

\begin{tabular}{|c|c|c|c|c|c|c|}
\hline Amostra & $\mathrm{T}_{\mathrm{eb}, \exp } / \mathrm{K}$ & $\mathrm{T}_{\mathrm{eb}, \mathrm{lit}} / \mathrm{K}^{*}$ & $\Delta(\%)$ & $\Delta_{\text {vap }} S_{\text {,exp }} / \mathrm{J} \mathrm{mol}^{-1} \mathrm{~K}^{-1}$ & $\Delta_{v a p} S_{\mathrm{lit}} / \mathrm{J} \mathrm{mol}^{-1} \mathrm{~K}^{-1 *}$ & $\Delta(\%)$ \\
\hline Éter etílico & 307,6 & 307,7 & $<0,1$ & $91,0 \pm 1,2$ & 93,9 & 3,1 \\
\hline Acetona & 322,0 & 329,2 & 2,2 & $96,4 \pm 1,4$ & 94,8 & $-1,7$ \\
\hline Etanol & 343,5 & 351,5 & 2,3 & $117,0 \pm 2,6$ & 117,9 & $-0,8$ \\
\hline Hexano & 325,2 & 342,2 & 5,0 & $79,2 \pm 3,6$ & 92,1 & 14 \\
\hline
\end{tabular}

*Lide, 1996 (valores calculados a partir dos valores de $\Delta_{v a p} H_{\text {lit }}$ da tabela anterior).

Os valores de $\Delta_{\text {vap }} H$ obtidos experimentalmente são bastante próximos daqueles da literatura, apresentando pequena diferença. A exceção é para o hexano, cujo valor obtido foi $14 \%$ menor do que o previsto. Deve-se considerar nesse caso que o composto normalmente se apresenta bastante contaminado com homólogos, de massas moleculares bastante próximas, e/ou com seus isômeros constitucionais, o que dificulta sua obtenção em estado mais puro.

Os valores de entalpia de vaporização das substâncias podem ser explicados por conta das interações intermoleculares e das massas moleculares de seus componentes. À exceção do hexano, todos os demais apresentam momento de dipolo permanente. Destes, o etanol tem momento dipolar mais intenso, seguido pela acetona e pelo éter etílico. Ressalte-se ainda que o primeiro exibe forte interação intermolecular, devido às ligações de hidrogênio. Dessa forma, justificam-se os valores de entalpia de vaporização dos mesmos. Quanto ao hexano, o valor se situa próximo àquele do éter etílico. Neste caso, o valor pode ser justificado não pelas forças de dipolo permanente (inexistentes nesse caso), mas pela intensidade das forças de dipolo momentâneo/dipolo induzido, maiores no hexano do que nos demais compostos, haja vista sua maior superfície.

A partir das retas experimentais obtidas para cada substância, é possível obter as temperaturas normais de ebulição e, por extensão, os valores de entropia normal de vaporização (Tabela 3) a partir da Equação 39

$$
\Delta_{v a p} S=\frac{\Delta_{v a p} H}{T_{e b}}
$$

As diferenças percentuais entre os pontos de ebulição obtidos no laboratório e os da literatura (e, por extensão, os valores de entropia de vaporização) podem ser consideradas satisfatórias, dadas as condições de trabalho em que foram determinados (uso dos compostos diretamente, sem purificação prévia, ausência de calibração e de correção da dilatação da coluna de mercúrio do termômetro).

Quanto às entropias de vaporização, deve-se atentar que os valores foram bem próximos daqueles previstos na literatura, à exceção do hexano. Em todos os casos, os valores são relativamente afastados do valor previsto pela regra de Trouton $\left(85 \mathrm{~J} \mathrm{~K}^{-1} \mathrm{~mol}^{-1}\right)$, devido às associações intermoleculares já discutidas. Nota-se em particular, o maior afastamento por parte do etanol, reflexo da importância das ligações intermoleculares neste caso.

Embora seja possível explicar os valores de entalpia de vaporização em função das interações intermoleculares e características das moléculas (superfície, massa molecular e geometria/momentos de dipolo), o conjunto de compostos examinados não permite que estas correlações sejam assumidas com todo rigor. Isso porque os compostos foram escolhidos em função de seu baixo custo e maior disponibilidade. Deve-se, no entanto, lembrar que o objetivo deste trabalho não é o de buscar as regularidades existentes entre características estruturais das moléculas e suas interações com o valor da entalpia de vaporização, mas sim o de proporcionar aos docentes um experimento que permite explorar o uso da Equação de Clapeyron na obtenção do valor de uma propriedade termodinâmica em laborató- rios de físico-química experimental, o que de modo algum exclui a possibilidade de seu uso em conjunto com as práticas didáticas dos professores de química orgânica teórica ou experimental.

Dessa maneira, a utilização de um aparato simples como o isoteniscópio permite a determinação da pressão de vapor de líquidos que, quando medida a várias temperaturas, permite calcular a entalpia e entropia de vaporização destes (medidas macroscópicas), propiciando-se discussões a respeito das interações moleculares (em nível microscópico).

\section{AGRADECIMENTOS}

Ao CNPq (proc. no. 400874/06-7) pelo financiamento de parte da execução deste trabalho, via projeto de Pesquisa em Ensino.

\section{REFERÊNCIAS E NOTAS}

1. Atkins, P.; Paula, J.; Físico-Química, $8^{\mathrm{a}}$ ed., LTC: Rio de Janeiro, 2008, vol. 1.

2. Castellan, G.; Fundamentos de Físico-Química, LTC: Rio de Janeiro, 1986.

3. Myrdal, P. B.; Krzyzaniak, J. F.; Yalkowsky, S. H.; Ind. Eng. Chem. Res. 1996, 35, 1788.

4. Lide, D. R.; Handbook of Chemistry and Physics, $77^{\text {th }}$ ed., CRC: New York, 1996.

5. Shoemaker, D. P.; Garland, C. W.; Experiments in physical chemistry, $4^{\text {th }}$ ed., Mc Graw-Hill Book Company: NewYork, 1981.

6. Rangel, R. N.; Práticas de Físico-Química, $2^{\mathrm{a}}$ ed., Edgard Blucher: São Paulo, 1998.

7. Kirk, G. S.; Raven, J. E.; Schofield, M.; Os Filósofos Pré-socráticos, $4^{\mathrm{a}}$ ed., Calouste Gulbenkian: Lisboa, 1994, p. 89.

8. Aristotle; On the Heavens, trad. de W. C. K. Guthrie; Loeb Classical Library, Harvard University Press: Massachusetts, 2006, vol. 338, p. 325

9. Wisniak, J.; J. Phase Equilibria 2001, 22, 622.

10. Avogadro, C. A.; Opere Scelte Pubblicate dalla R. Academia delle Scienze di Torino, Editrice Torinese: Torino, 1911.

11. Weast, R. C., ed.; CRC Handbook of Chemistry and Physics, 52 ${ }^{\text {th }}$ ed.; CRC: Cleveland, 1971.

12. Bertrand, J.; Thermodynamique, Gauthier-Villars: Paris, 1887.

13. Uma lista de autores que aborda a Equação de Clausius ou de ClausiusClapeyron a partir de considerações da igualdade dos potenciais químicos e da dependência da entropia com as variações de pressão e temperatura é dada a seguir: Moore, W. J.; Físico-química, EDUSP/Edgar Blucher: São Paulo, 1976, vol. 1; Tinoco, I.; Sauer, K.; Wang, J.; Physical Chemistry: principles and applications in biological science, $3^{\text {rd }}$ ed., Prentice-Hall: New Jersey, 1995; Alberty, R. A.; Physical Chemistry, $7^{\text {th }}$ ed., John Wiley: New York, 1987; Eggers, D. F.; Gregory, N. W.; Halsey, G. D.; Rabinovitch, B. S.; Physical Chemistry, Wiley Interscience: New York, 1964; Pilla, L.; Físico-química, LTC: Rio de Janeiro, 1979, vol. 1; Rowlinson, J. S.; Liquids and Liquid Mixtures, Butterworths: London, 1959; Chagas, A. P.; Termodinâmica Química, Ed. da UNICAMP: Campinas, 1999; Castellan, op. cit..

14. Lee, J. F.; Sears, F. W.; Termodinâmica, LTC: Rio de Janeiro, 1969.

15. Novak, I.; Chemical Educator 2002, 7, 347.

16. Armarego, W. L. F.; Perrin, D. D.; Purification of Laboratory Chemicals, $4^{\text {th }}$ ed., Butterworth-Heinemann: Oxford, 1998. 